\title{
Epimutation profiling in Beckwith-Wiedemann syndrome: relationship with assisted reproductive technology
}

\author{
Louise Tee ${ }^{1}$, Derek HK Lim,2, Renuka P Dias ${ }^{1,3}$, Marie-Odile Baudement ${ }^{1}$, Amy A Slater ${ }^{1}$, Gail Kirby ${ }^{1,2}$,
} Tom Hancocks ${ }^{1}$, Helen Stewart ${ }^{4}$, Carol Hardy ${ }^{2}$, Fiona Macdonald ${ }^{2}$ and Eamonn R Maher ${ }^{1,2,5^{*}}$

\begin{abstract}
Background: Beckwith-Wiedemann syndrome (BWS) is a congenital overgrowth disorder associated with abnormalities in 11 p15.5 imprinted genes. The most common cause is loss of methylation (epimutation) at the imprinting control centre 2 (IC2/KvDMR1). Most IC2 epimutations occur sporadically but an association with conception after assisted reproductive technologies (ART) has been reported. A subgroup of IC2 epimutation cases also harbour epimutations at other imprinting centres (ICS) outside of 11 p15.5. We have investigated the relationship between these multiple epimutation cases (ME+), history of ART and clinical phenotype in a cohort of 187 BWS IC2 epimutation patients.
\end{abstract}

Results: Methylation analysis at PLAGL1, MEST and IGF2R ICS demonstrated an over-representation of patients with abnormally low methylation ( $8.5 \%, 12 \%$ and $6 \%$ respectively). At IGF2R some patients (2\%) had gain of methylation but this was also detected in controls. Though there were no significant correlations between the methylation index (Mls) at the three ICs tested, a subset of patients appeared to be susceptible to multiple epimutations (ME+) and $21.2 \%$ of $\mathrm{ME}+$ patients had been conceived by ART compared to $4.5 \%$ ( $P=0.0033$ ) without additional epimutations. Methylation array profiling (Illumina Goldengate ${ }^{\oplus}$ ) of patients and controls (excluding 11 p15.5 loci) demonstrated significant differences between patients and controls. No significant associations were found between aspects of the BWS phenotype and individual epimutations but we describe a case presenting with a post-ART BWS-like phenotype in which molecular analysis demonstrated loss of paternal allele methylation at the 11 p15.5 IC1 locus (IC1 regulates imprinting of IGF2 and H19). Loss of paternal allele methylation at the IC1 is the molecular finding associated with Silver-Russell syndrome whereas BWS is associated with gain of maternal allele methylation at IC1. Further analysis demonstrated epimutations at PLAGL1 and MEST consistent with the hypothesis that the presence of multiple epimutations may be of clinical relevance.

Conclusions: These findings suggest that the ME + subgroup of BWS patients are preferentially, but not exclusively, associated with a history of ART and that, though at present, there are no clear epigenotype-phenotype correlations for ME + BWS patients, non-11 p15.5 IC epimutations can influence clinical phenotype.

Keywords: Beckwith-Wiedemann syndrome, Assisted reproductive technology, Imprinting, Genetics

\footnotetext{
* Correspondence: erm1000@medschl.cam.ac.uk

${ }^{1}$ Centre for Rare Diseases and Personalised Medicine, School of Clinical and Experimental Medicine, College of Medical and Dental Sciences, University of Birmingham, Edgbaston, Birmingham B15 2TT, UK

${ }^{2}$ West Midlands Regional Genetics Service, Birmingham Women's Hospital, Edgbaston, Birmingham B15 2TG, UK

Full list of author information is available at the end of the article
} 


\section{Background}

Genomic imprinting is a form of epigenetic control of gene expression in which one allele of a gene is preferentially expressed according to the parent-of-origin of the allele [1]. Only a minority of human genes (approximately 100) are imprinted but many of those identified to date have been implicated in prenatal growth and development [2,3]. A notable feature of imprinted genes is that they tend to be found in clusters and not randomly distributed across the genome. A number of different mechanisms (for example, DNA methylation, chromatin modification and expression of large noncoding RNAs) have been implicated in the establishment and maintenance of genomic imprinting and a key role is played by imprinting control centres (ICs), which may regulate the imprinting of several genes [1]. ICs are often coincident with differentially methylated regions (DMRs) at which the methylation status of the CpG islands differ according to the parent-of-origin. Deletion or abnormal methylation (an epimutation) at these sites may cause abnormal imprinting within the imprinted gene cluster. For example, gain of methylation (GOM) at the normally unmethylated maternal allele of the chromosome 11p15.5 IC1 DMR is associated with bi-allelic expression of the paternally expressed growth promoter IGF2 and is associated with the Beckwith-Wiedemann congenital overgrowth syndrome (BWS) [4]. In contrast, loss of methylation (LOM) of the paternal allele at the same IC is associated with loss of paternal allele IGF2 expression and the phenotype of Silver-Russell syndrome (SRS), which is characterised by pre- and postnatal growth restriction [5].

Epimutations at the IC1 DMR occur in 5 to $10 \%$ of children with BWS but a more common cause of this disorder is loss of maternal allele methylation at a DMR (KvDMR1) associated with a second 11p15.5 IC (IC2). Thus, IC2 epimutations account for approximately $50 \%$ of all cases of BWS [6]. IC2 epimutations are associated with loss of expression of the imprinted maternally expressed growth suppressor CDKN1C (loss of function mutations in this gene can also cause BWS). Interestingly, a subset of BWS patients with IC2 epimutations also harbour epimutations at other imprinted gene cluster DMRs outside of 11p15.5 (for example, PLAGL1 and MEST (PEG1) on chromosomes 6 and 7 respectively) $[7,8]$. The clinical consequences and aetiology of these multiple epimutations are mostly unclear, though in a minority of cases a rare genetic cause may be identified and in others there is an association with a history of conception by assisted reproductive technology (ART) [9-11]. To further investigate the potential significance of multiple epimutation epigenotypes we undertook methylation assays and epigenotype-phenotype correlations in patients with BWS and IC2 epimutations, paying particular attention to possible associations between epimutations and ART.

\section{Methods}

\section{Patients}

Children and adults $(\mathrm{n}=187)$ with a clinical and molecular diagnosis of BWS were studied for methylation status at one or more imprinted loci outside $11 \mathrm{p} 15.5$ (PLAGL1, MEST and IGF2R DMRs). Clinical information was collected using a standard questionnaire. Fourteen patients had been conceived after ART by in vitro fertilization (IVF) or intracytoplasmic sperm injection (ICSI). Written informed consent was obtained and ethical approval was obtained from South Birmingham Research Ethics committee.

\section{Molecular analysis}

DNA was extracted from peripheral blood lymphocytes by standard procedures. Previously all BWS patients had been shown to have loss of methylation at KvDMR1 (11p15.5 IC2) without evidence of uniparental disomy or copy number abnormalities by methylation-specific-multiplex ligation-dependent probe amplification for genetic disease and genomic imprinting research (MS-MLPA) analysis (MRC-Holland, Amsterdam, The Netherlands). Anonymised DNA samples from healthy individuals without an imprinting disorder were used as controls for the methylation studies. Grandparental origin of the maternally transmitted IC2 region was established by genotyping microsatellite polymorphisms (TH, D11S1318 and D11S1984) as described previously [12].

\section{Methylation at PLAG1, MEST and IGF2R DMRs}

For differential digestion of DNA for methylation analysis, 0.5 ug of genomic DNA was treated using the Epitect Methyl DNA Restriction Kit (SA Biosciences, Qiagen Ltd, West Sussex, UK 335451) as per instructions. Predesigned qPCR assays for MEST (SA Biosciences, MePH10851) and PLAGL1 (SA Biosciences, MePH285522$1 \mathrm{~A})$ were used. For the CpG island in intron 2 of IGF2R, a custom quantitative PCR (qPCR) assay was designed by SA Biosciences. Real-time PCR was performed on the BioRad iCycler thermal cycler using the SensiMix Sybr with fluorescein kit (Bioline, London, UK, QT615-02) conditions as per SA Biosciences protocol.

Data analysis was performed using software supplied on the SA Biosciences website, whereby control values obtained from the real-time PCRs were pasted into an Excel $^{\circ}$-based template. The template automatically calculated and reported the percentage of DNA hypermethylated and unmethylated fractions. To establish normal ranges and therefore, a level at which LOM could be assumed for each of the assays tested, a series of normal control samples were run. Methylation index (MI, 
percentage hypermethylation) values obtained were used for calculations. For PLAGL1 DMR MI results were obtained for 20 control samples. The average MI was $47.33 \%$ and the range was 36.38 to $55.08 \%$ with SD of 4.91. Three times the SD from the average MI included all control values and set the lower threshold for LOM at $32.6 \%$. For MEST DMR results were obtained for 11 control samples. The average MI was $47.83 \%$, range was 32.54 to $55.48 \%$ and SD $7.73 \%$. Two times the SD from the average $\mathrm{MI}$ included all control values and set the lower threshold for LOM at $32.37 \%$. For IGF2R DMR results were obtained for 13 control samples. The median MI was $50.3 \%$ and the average MI was $57.05 \%$ with a range of 41.1 to $97.7 \%$. D'Agostino-Pearson testing for normality of the distribution rejected normality due to an outlier value of 97.7\%. Excluding the outlier, the median was $50.15 \%$, mean $53.66 \%$ (range 41.1 to 74.99 ) and SD was $9.5 \%$. Two times the SD from the mean MI set the lower threshold for LOM at $34.66 \%$.

Limited amounts of available DNA meant that it was not possible to obtain a methylation index (MI) for each of the three tested loci in all patients. A value for the $\mathrm{MI} \geq 1$ locus was obtained for 187 patients but MI values at all three loci were obtained for only 150 samples (11 samples failed for PLAGL1 MI (5 for both PLAGL1 and IGF2R DMRs): 20 failed for MEST DMR ( 9 for both MEST and IGF2R DMRs) and 6 samples failed for IGF2R DMR only).

\section{Illumina Goldengate methylation analysis}

DNA samples of $0.5 \mu \mathrm{g}$ were treated with sodium bisulphite using the EZ DNA methylation Gold kit (Zymo Research, Irvine, USA), and the bisulphite-treated DNA was applied to an Illumina bead array (10) using the Illumina Goldengate Methylation Cancer Panel (http://support.llumina.com/ downloads/goldengate_methylation_cancer_panel_product_ files.ilmn) (performed at the Wellcome Trust Centre for Human Genetics, University of Oxford, UK), which has been described and validated previously [13]. Hierarchical cluster analysis was performed using Illumina BeadStudio software (http://res.illumina.com/documents/products/datasheets/datasheet_beadstudio.pdf) with the Euclidean algorithm (see http://res.illumina.com/documents/products/ technotes/technote_beadstudio_normalization.pdf) and used to create heatmaps and associated dendrograms.

\section{Results}

Methylation at PLAGL1, MEST and IGF2R DMRs in patients with BWS and 11p15.5 IC2 epimutations

Mean (+ SD) MI at the PLAGL1 DMR was $47 \% \pm 10.56$ (median 50\%, range 0.0055 to 67.13 ) in BWS patients with IC2 epimutations $(\mathrm{n}=176)$. In comparison to normal controls $(n=20)$, there was an over-representation of low MIs in patient samples (Figure 1A) and the distribution of PLAGL1 DMR MIs did not conform to a

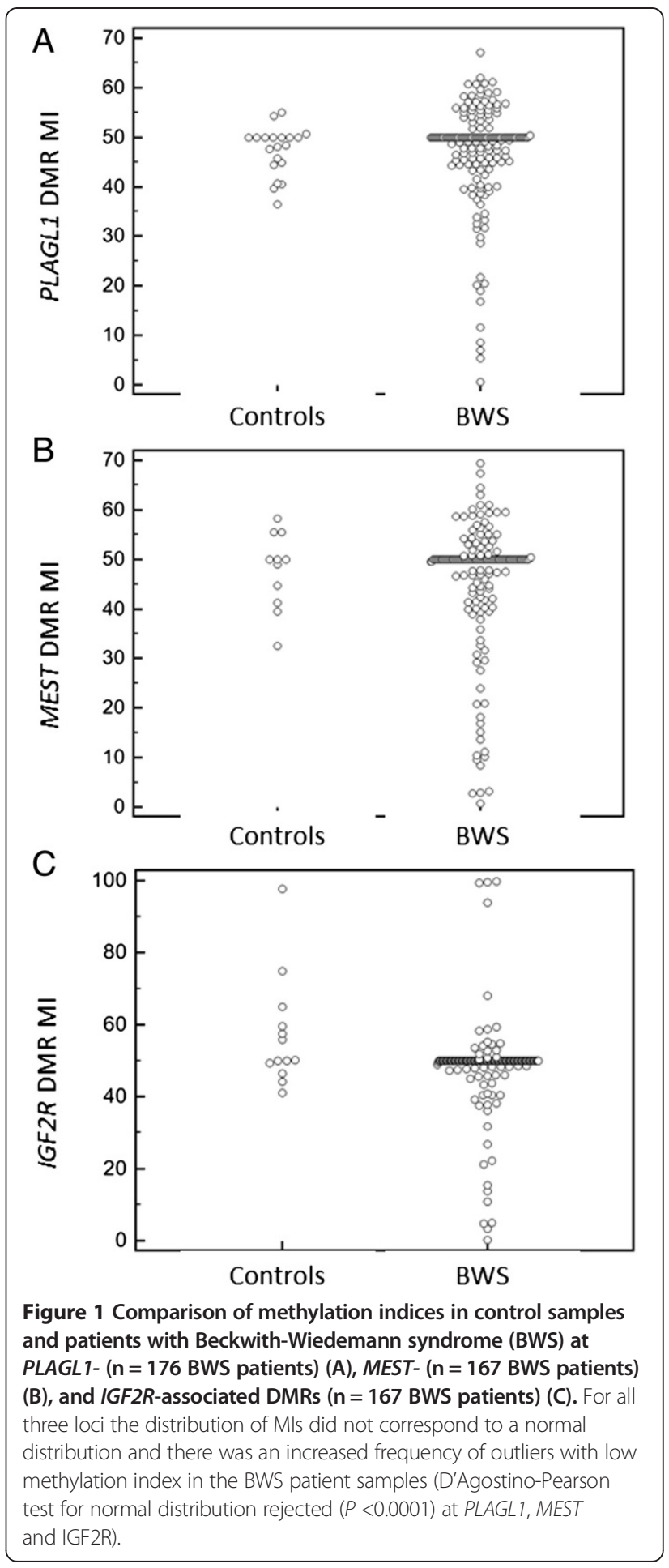

normal distribution (D'Agostino-Pearson test for normal distribution rejected at $P<0.0001$; coefficient of skewness, $P<0.0001)$. Thus, $15(8.5 \%)$ patients had an abnormally low MI (MI <33\%) at the PLAGL1 DMR (median 20.2\%, range 0.6 to $32.6 \%$ ). 
Mean (+ SD) MI at the MEST (also known as PEG1) DMR was $46.4 \%+12.54$ (median $50 \%$, range 0.0012 to 99.76) in BWS patients with IC2 epimutations $(n=167)$. In comparison to normal controls $(n=11)$, there was over-representation of low MIs in patient samples (Figure 1B) and the distribution of MEST DMR MIs did not conform to a normal distribution (D'Agostino-Pearson test for normal distribution rejected at $P<0.0001$ ) with significant evidence of kurtosis (coefficient of kurtosis 9.016; $P<0.0001$ ) (see Figure 1B). Twenty of the BWS patients $(12 \%)$ who were successfully tested had an abnormally low MI (MI <31\%) at the MEST DMR (median MI $14.3 \%$, range 0.7 to $30.8 \%)$.

Mean (+ SD) MI at the IGF2R DMR was $45.7 \%+12.98$ (median 50\%, range 0.0072 to 69.37 ) in BWS patients with IC2 epimutations $(\mathrm{n}=167)$. IGF2 DMR MI was measured in 13 control samples. The distribution of IGF2R DMR MIs did not conform to a normal distribution (D'Agostino-Pearson test for normal distribution rejected at $P<0.0001$; coefficient of skewness -1.815 ; $P<0.0001$ ) (see Figure 1C). However, unlike the PLAGL1 and MEST DMR MIs, both low and high MIs were detected: 10 patients $(6 \%)$ had loss of methylation (MI <31\%) at the IGF2R DMR (median 12.3\%, range 0.1 to $26.8 \%)$ and 4 patients (2\%) had increased methylation (MI >90\%) (median 99.5\%, range 93.9 to $99.8 \%$ ), although an individual with high methylation was also detected in control samples.

Though there were was no significant correlations between the MIs at the PLAGL1, MEST and IGF2R DMRs (Spearman rank correlation analysis), a subset of patients with BWS appeared to be susceptible to multiple epimutations. Thus, of the cases for whom data were available at all three non-11p15.5 IC DMRs, 81.7\% did not have hypomethylation at the three tested loci, $12.4 \%$ had hypomethylation at one locus, $3.9 \%$ at two loci and $2 \%$ had hypomethylation at all three IC DMRs tested. Thus the frequency of hypomethylation at MEST and/or IGF2R1 DMRs was higher in those with than those without PLAGL1 DMR hypomethylation $(41.6 \%$ versus $10.5 \% ; P=0.009)$. Similarly the frequency of hypomethylation at PLAGL1 and/or IGF2R1 DMRs was higher in those with than those without MEST DMR hypomethylation $(55.6 \%$ versus $7.4 \% ; P=0.00003)$ and the frequency of hypomethylation at PLAGL1 and/or MEST DMRs was higher in those with than those without IGF2R DMR hypomethylation (70\% versus $13.3 \%$; $P=0.00016)$.

\section{Multiple epimutation epigenotype and assisted reproductive technologies}

Of 187 BWS patients tested for additional epimutations, 14 had been conceived by ART: 7 of 33 BWS patients (21.2\%) with LOM at one or more of the tested DMRs
(PLAGL1, MEST and IGF2R) had been conceived by IVF or ICSI compared to 7 of $154(4.5 \%)(P=0.0033)$ without evidence of LOM at one or more of the tested DMRs. Thus $50 \%$ (7 of 14) of BWS patients with an IC2 epimutation, who were conceived after ART, displayed a multiple epimutation epigenotype compared to 15\% (26/ 173) of naturally conceived patients. Of the 14 ART cases, 9 were conceived by IVF and 5 by ICSI: 5 of 9 patients conceived by IVF and 2 of 5 patients conceived by ICSI had a multiple epimutation epigenotype $(P=1.0)$. None of the four BWS patients with increased methylation at the IGF2R DMR were conceived by IVF or ICSI and none demonstrated LOM at PLAGL1 or MEST DMRs.

\section{ART and multiple epimutation genotype and clinical phenotype}

The frequencies of specific clinical features among the cohort of individuals with BWS and IC2 epimutations were: macroglossia (94\%), ear creases or pits $(80 \%)$, neonatal hypoglycaemia (58\%), facial naevus flammeus (54\%), exomphalos (52\%), umbilical hernia (34.8\%), hemihypertrophy (28\%), cardiovascular abnormality (18\%), prenatal pre-eclampsia (15\%), developmental delay (9\%) and cleft palate (2\%). Only one patient had developed an embryonal tumour (a hepatoblastoma). No significant associations were detected between the frequency of any of these specific clinical features and the presence of multiple epimutations (loss of methylation at PLAGL1, MEST or IGF2R DMRs).

Comparison of gestation-adjusted birth weight SD from mean in patients with $(+1.4 \pm 1.47$ (mean $\pm \mathrm{SD})$; range -2.19 to +3.31$)$ and without $(+1.31 \pm 1.32 ;-3.56$ to +4.75$)$ multiple epimutations demonstrated no significant difference $(t=-0.26, P=0.8)$. There were no significant correlations between mean gestation-adjusted birth weight SD and MI index at PLAGL1 (Spearman's coefficient of rank correlation (rho) $=0.084 ; P=0.37$ ), MEST (rho $=-0.037 ; P=0.7)$ and $I G F 2 R($ rho $=-0.083 ; P=0.38)$.

\section{Methylation profiling by Illumina Goldengate CpG methylation assay}

The Illumina GoldenGate Methylation Cancer Panel I array provides quantitative $\mathrm{CpG}$ methylation data at 1,505 individual CpG dinucleotides associated with 807 human genes. This platform was used to profile the peripheral blood methylation patterns of BWS patients and controls. We initially excluded $638 \mathrm{CpG}$ sites from further analysis because (a) they were on the $\mathrm{X}$ chromosome genes or were in the 11p15.5-imprinted gene cluster and (b) the CpG methylation results were poorly replicated in controls ( $>10 \%$ methylation difference) (see Additional file 1: Table S1). Euclidean cluster analysis was performed for (a) all imprinted $\mathrm{CpG}$ sites that mapped 
outside 11p15.5 $(\mathrm{n}=53)$ and (b) all non-imprinted CpG sites $(n=865)$. Analysis of the imprinted gene $\mathrm{CpG}$ methylation data for BWS patients and controls demonstrated two principal clusters (Figure 2A and Additional file 2: Figure S1). Cluster 1 contained 23 BWS patients and one control whereas cluster 2 contained 27 BWS patients and 15 controls $(P=0.0058)$. The four BWS patients whose methylation profile was most different to the control samples were all conceived by ART. However, of the 10 BWS patients who had been conceived by ART, 5 were in cluster 1 and 5 in cluster 2 , and overall there was no significant association between ART and the non-11p15.5-imprinted gene methylation profile. Significant clustering of controls versus BWS was seen when non-imprinted $\mathrm{CpG}$ sites were analysed $(P=0.0402)$ (Figure $2 \mathrm{~B}$ and Additional file 2: Figure S2) but there were no significant differences between ART-conceived and naturally conceived BWS patients at non-imprinted CpG sites $(P=0.3308)$. Details of the absolute MI for each $\mathrm{CpG}$ analysed for clustering analysis are given in Additional file 1: Table S1.

\section{Grandparental origin of IC2 epimutations}

We investigated the grandpaternal origin of the maternally inherited IC2 region in individuals with loss of methylation at IC2. A bias in the grandpaternal origin would provide clues to the possible mechanism for loss of IC2 methylation. The grandparental origin of the maternal KvDMR1 allele that had lost the correct methylation imprint was determined in 10 patients by genotyping with closely linked microsatellite markers. In seven patients the maternally inherited IC2 allele had been derived from the maternal grandmother and in three patients from the maternal grandfather. There was no significant association between the grandparental origin and presence or absence of additional epimutations at PLAGL1, MEST or IGF2R DMRs $(P=1.0)$ or a history of IVF/ICSI $(P=1.0)$.

\section{IC1 hypomethylation in BWS: case report}

The proband is a male child born at 41 weeks gestation following an ICSI conception. Birth weight was on the 25th centile and no neonatal hypoglycaemia was reported. However, at age 5 weeks he presented to hospital with a history of breathing problems with breathholding spells and apnoea. Otolaryngological assessment identified severe macroglossia as contributing to his apnoea and he required a tracheostomy at age 2 months. A referral for clinical genetics assessment was made and clinical examination identified severe macroglossia, postnatal overgrowth (75th centile for weight and 91st centile for head circumference at age 16 weeks, and subsequently 91st centile for weight at age 2 years and 99th centile for weight, 95th centile for head circumference and just below 50th centile for height at age 4 years), ear lobe creases, facial naevus flammeus, umbilical hernia and discrepancy in leg size. No organomegaly was detected on ultrasound scans or normal echocardiogram. A clinical diagnosis of BWS was made and MS-MLPA analysis (MRC Holland) was performed to look for epimutations associated with BWS (IC2 (KvDMR1) loss of methylation or IC1 (H19-IGF2 DMR) hypermethylation). Unexpectedly, IC1 (H19-IGF2 DMR) hypomethylation was detected. No chromosomal copy number abnormalities were detected. In view of the discrepancy between the clinical phenotype of BWS and the molecular findings of SRS, additional methylation analysis was performed at the PLAGL1 and MEST DMRs and loss of methylation was detected at both loci.

\section{Discussion}

We profiled a large series of patients with BWS and an IC2/KvDMR1 epimutation to identify additional epimutations at DMRs outside of 11p15.5. We found that the multiple epimutation epigenotype $(\mathrm{ME}+)$ occurred in a subset of patients and was associated with a history of ART. However, the multiple epimutation epigenotype was not restricted to ART-conceived patients and was also found in a subset of non-ART BWS patients with IC2 epimutations. Imprinted gene cluster DMRs may act as boundary elements and/or regulate the expression of imprinted non-coding RNAs (for example, H19, KCN1OT and Air) and the methylation status of the DMR is critical to their normal function. For example, the insulating CTCF zinc finger protein normally binds to the unmethylated maternal allele of IC1 (H19) DMR on 11p1.5.5, enabling downstream enhancers to preferentially interact with the H19 promoter. Methylation of the DMR on the paternal allele (containing the CTCF binding region) prevents CTCF binding and allows the enhancers to interact with the IGF2 promoter instead [14,15]. Conversely, loss of IC1 methylation on the paternal allele (as seen in SRS) is associated with loss of paternal allele IGF2 expression [5].

Most cases of imprinting disorders associated with a DMR epimutation occur sporadically. In a few cases, apparent epimutations result from a deletion or mutation in the IC (that is, an in cis mechanism) and some cases can occur via trans-mechanism leading to abnormal DNA methylation, often at multiple imprinted loci. Thus, patients with mutations in ZFP57 (which is involved in the establishment and maintenance of genomic imprinting) presenting with prenatal growth failure and transient neonatal diabetes mellitus (TNDM, OMIM 601410) showed loss of maternal methylation at the PLAGL1 DMR and variable loss of maternal methylation at GRB10, PEG3, NESPAS and MEST [16]. Overall 


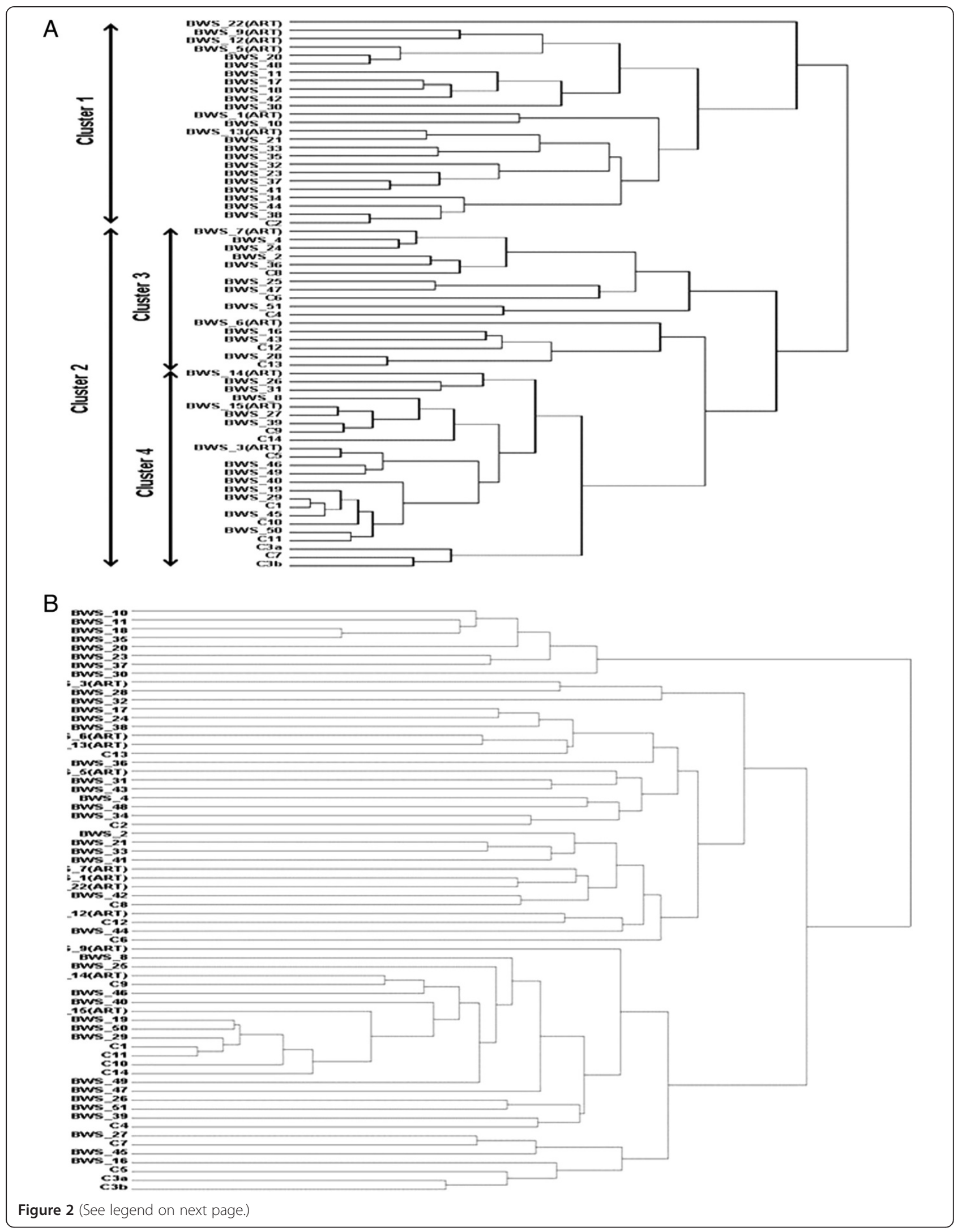


(See figure on previous page.)

Figure 2 Clustering dendrograms of Beckwith-Wiedemann syndrome (BWS) patients and controls (samples were assigned arbitrary numbers) according to methylation state of (A) all imprinted genes (excluding H19 and KvDMR1) and (B) all non-imprinted genes. *BWS indicates samples from patients with BWS and C indicates control samples. Suffix (ART) denotes BWS patients conceived with ART. (A) Unsupervised analysis of cluster 1 versus cluster 2 showed significant grouping of controls versus BWS patients ( $P=0.0054)$. No significant clustering was detected of (i) BWS patients conceived via ART compared to BWS patients conceived naturally $(P=0.3082)$ and (ii) controls versus BWS between cluster 3 and $4(P=0.52)$. (B) Analysis of methylation status of CpGs associated with non-imprinted genes showed significant clustering of controls versus BWS $(P=0.0402)$ but no significant clustering of BWS conceived by ART compared to BWS patients conceived naturally $(P=0.3308)$.

however, mutations in ZFP57 and other trans-acting factors implicated in the establishment of genomic imprinting are rare causes of various imprinting disorder phenotypes [16-18]. In addition to genetic causes, environmental factors may be associated with epimutations in a subset of patients. Thus, we and others have found an excess of being conceived by ART among children with sporadic BWS $[9,11]$. Further studies have demonstrated that this finding reflects an association between postART BWS and IC2 epimutations (IC2 epimutations are found in $>90 \%$ of post-ART BWS compared to approximately $50 \%$ of all cases) $[10,19]$. Though the relative risk of BWS (and possibly other imprinting disorders) is increased after ART, the absolute risk of having a child with an imprinting disorder for an individual ARTpregnancy appears to be small $(<0.1 \%)$ [20-22]. Nevertheless, there is much interest in any association between ART and imprinting disorders as it would lend support to the hypothesis that congenital anomalies and growth restriction in ART-conceived children could be explained, at least in part, by epimutations [10].

Previously it was reported that about $25 \%$ of BWS patients with IC2 epimutations were also reported to have additional epimutations at non-11p15.5 IC DMRs $[7,8]$. Other imprinting disorders such as SRS and pseudohypoparathyroidism (PHP type 1B) have also shown an $\mathrm{ME}+$ epigenotype [23-26]. However, in general the aetiology and clinical significance of $\mathrm{ME}+$ cases has been unclear. Although in a previous study of 55 patients with BWS and IC2 epimutations we found an association between BWS ME + cases and a history of ART, such an association has not been detected consistently [26,27]. Possible reasons for these differences might include inadequately powered studies or differences in ART protocols or DNA methylation detection assays. We therefore undertook methylation profiling a larger series of BWS patients at three specific IC DMRs (PLAGL1, MEST and $I G F 2 R$ ) that have been commonly reported to show aberrant methylation in imprinting disorder patients with multiple epimutations.

Loss of maternal allele methylation at the PLAGL1 DMR at 6q24-25 is the primary epimutation seen in TNDM but has also been reported to be hypomethylated in $2-9 \%$ of BWS patients and in SRS [7,8,10,12,23,24].
Interestingly whereas BWS is characterised by prenatal overgrowth and frequent neonatal hypoglycaemia, TNDM is characterised by intra-uterine growth failure and lack of subcutaneous fat and neonatal hyperglycaemia [28,29]. Up to $10 \%$ of patients with SRS have maternal uniparental disomy of chromosome 7 (mUPD7) and a small number of cases will have chromosome 7 duplications [30]. The MEST DMR maps to 7q32 and in mice loss of the paternal allele leads to fetal growth failure [31]. Hence MEST has been extensively investigated as a potential candidate gene for SRS and although the precise role of this locus in SRSassociated growth restriction is still unclear, MEST DMR epimutations have been described in patients with BWS (approximately 8\%), PHP1B (10\%) and ME + SRS [7,25]. The insulin-like growth factor 2 receptor (IGF2R) inhibits IGF2-induced overgrowth by promoting clearance of IGF2 from the circulation [32]. In mice Igf $2 r$ is imprinted and maternally expressed but the evidence that IGF2R is imprinted in humans is less strong, though it may exhibit polymorphic imprinting [33]. In theory, gain of methylation at the IGF2R DMR would increase IGF2R expression and inhibit fetal growth whereas loss of methylation would promote overgrowth [34,35].

We found that LOM at PLAGL1 and MEST DMRs occurred in a minority of BWS patients at a frequency that was similar to that in other studies [7]. As reported by others, we found that both LOM and GOM could occur at IGF2R DMR [36]. IGF2R hypermethylation has been reported in patients with growth retardation with and without SRS and we found IGF2R DMR hypermethylation in four BWS patients and a control individual. Though we did not find any overall correlations between methylation levels at PLAGL1, MEST and IGF2R DMRs, there was a tendency for hypomethylation at these loci to cluster in a small subset of patients. However, there was no evidence that patients with IGF2R hypermethylation were more susceptible to loss of methylation at PLAGL1 or MEST DMRs. As found in our previous smaller study, ART-conceived children were significantly over-represented among BWS ME + patients but more extensive methylation profiling using the $\mathrm{CpG}$ methylation array did not clearly differentiate between post-ART and naturally conceived BWS patients. Previously methylation profiling at imprinted loci in ART-conceived children 
without imprinting disorders has shown no generalised tendency to methylation changes [37]. We investigated a cohort with IC2 epimutations and though it could be argued that such cases might have been susceptible to ART-associated imprinting errors, only a minority had a $\mathrm{ME}+$ epigenotype. At this stage it is not known whether this subset of $\mathrm{ME}+$ patients are genetically predisposed to epimutations (for example, in response to environmental factors such as ART) or whether there are specific environmental factors implicated (for example, precise embryo culture conditions in post-ART cases).

We investigated whether the loss of maternal allele KvDMR1 methylation (a paternal epigenotype pattern) in BWS IC2 epimutation cases was associated with the grandparental origin of the allele (hypothesising that the LOM might result from a failure to reset the imprinting on the grandpaternal chromosome). We found no evidence of bias in grandparental origin suggesting that the imprinting defect occurred after erasure of the parental imprint and the defective imprinting resulted from disordered establishment or maintenance of the maternal allele imprint (which might be after fertilization). Interestingly, Buiting et al. reported that in patients with Angelman syndrome (AS) and 15q11 IC epimutations (loss of maternal allele methylation), there was no bias in grandparental origin of the chromosome carrying the abnormal imprint, but in Prader-Willi syndrome patients with no IC deletion, the paternal chromosome carrying an incorrect maternal imprint was preferentially derived from the paternal grandmother, raising the possibility that such cases result from a failure to erase the maternal imprint [36]. Both BWS and AS have been associated with ART and the suggestion that epimutations in these disorders might result from failure to establish or maintain germline imprints could fit with either of the major hypotheses for the association of imprinting disorders with ART, namely, (a) that infertility per se predisposes to abnormal imprinting and/or (b) that aspects of ART (for example, ovarian hyperstimulation, in vitro embryo culture etcetera) predispose to abnormal imprinting [20,38].

\section{Conclusions}

We did not detect any significant associations between loss (or gain) of methylation at PLAGL1, MEST and IGF2R DMRs and gestation-adjusted birth weight scores or specific clinical features of BWS and investigations in other cohorts have not detected consistent statistically significant clinical correlates of an $\mathrm{ME}+$ phenotype $[7,8]$. Though these findings could be interpreted as indicating that extra-11p15.5 epimutations might have no clinical significance, the case report of an ART-conceived child with clinically diagnosed BWS but loss of paternal allele methylation at the IC1 DMR (predicting a clinical diagnosis of SRS) and epimutations at extra-11p15.5 DMRs strongly suggests that there can be clinical significance. The failure to detect a clear relationship between epimutations at specific DMRs and a specific clinical phenotype may reflect a number of variables including, (a) the variability of degree of methylation loss at individual DMRs (consistent with mosaicism that might differ between tissues), (b) that a comprehensive analysis of all IC DMRs has not been undertaken (and it cannot be excluded that epimutations at currently unrecognised IC DMRs might exist) and (c) the complexity of functional interactions between imprinted genes (for example, PLAGL1 and IGF2 [39,40]). It was interesting that we found some evidence that there might be differences between BWS patients and controls at non-imprinted $\mathrm{CpG}$ sites. Further studies are required to confirm this observation. Analyses to uncover potential effects of specific aspects of an ME + epigenotype on the phenotypic expression of BWS are likely to require both comprehensive IC methylation profiling and multicentre collaboration to generate large cohorts of patients. Nevertheless, such a study would have relevance for BWS and other imprinting disorders and might also inform the potential role of epimutations in children conceived by ART.

\section{Additional files}

Additional file 1: Table S1. Absolute methylation indices for each of the analysed CpG on Illumina ${ }^{\oplus}$ GoldenGate Cancer Panel 1. Target ID (as per array output) is given. Red indicates imprinted CpGs. Methylation values given to three decimal places. Sample ID refers to patients with Beckwith-Wiedemann syndrome (BWS) and controls. The suffix (ART) indicates conception by assisted reproductive technologies.

Additional file 2: Figure S1. Analysis of the imprinted gene CpG methylation data for Beckwith-Wiedemann syndrome (BWS) patients and controls demonstrated two principal clusters (see Results for details). The colours indicate methylation levels, ranging from red (hypermethylated) to blue (hypomethylated). Figure S2. Significant clustering of controls versus BWS seen when methylation at non-imprinted CpG sites was analysed ( $P=0.0402$ ) (see Results for details). The colours indicate methylation levels, ranging from red (hypermethylated) to blue (hypomethylated).

\begin{abstract}
Abbreviations
AS: Angelman syndrome; ART: Assisted reproductive technologies; BWS: Beckwith-Wiedemann syndrome; IC: Imprinting centre; DMR: Differentially methylated region; GOM: Gain of methylation; ICSI: Intracytoplasmic sperm injection; IGF2R: Insulin-like growth factor 2 receptor; IVF: In vitro fertilization; LOM: Loss of methylation; MI: Methylation index; MS-MLPA: Methylation-specific-multiplex ligation-dependent probe amplification; qPCR: Quantitative polymerase chain reaction; SRS: Silver-Russell syndrome; TNDM: Transient neonatal diabetes mellitus.
\end{abstract}

\section{Competing interests}

The authors declare that they have no competing interests.

\section{Authors' contributions}

$L T, A S, M O, T H$ and $C H$ performed laboratory investigations; DL, GK, HS, FM and EM collected and interpreted clinical and molecular information; LT, RPD and EM performed statistical analysis and data interpretation; EM conceived the study and wrote the first draft of the manuscript. All authors critically revised the manuscript. All authors read and approved the final manuscript. 


\section{Acknowledgements}

We thank Action Medical Research (LT and EM) and Birmingham Children's Hospital, Newlife Foundation and the Child Growth Foundation (RPD) for funding. LT is funded by Action Medical Research; RPD is funded by Birmingham Children's Hospital with additional funding from Newlife Foundation and the Child Growth Foundation. The funding bodies had no involvement in the design, implementation or reporting of this research.

\section{Disclosure summary}

The authors have nothing to disclose.

\section{Author details}

${ }^{1}$ Centre for Rare Diseases and Personalised Medicine, School of Clinical and Experimental Medicine, College of Medical and Dental Sciences, University of Birmingham, Edgbaston, Birmingham B15 2TT, UK. ${ }^{2}$ West Midlands Regional Genetics Service, Birmingham Women's Hospital, Edgbaston, Birmingham B15 2TG, UK. 'Department of Endocrinology, Birmingham Children's Hospital, Steelhouse Lane, Birmingham B4 6NH, UK. ${ }^{4}$ Department of Clinical Genetics, Oxford Regional Genetics Service, Churchill Hospital, Oxford, UK.

${ }^{5}$ Department of Medical Genetics, University of Cambridge, Cambridge CB2 2QQ, UK.

Received: 8 July 2013 Accepted: 1 November 2013

Published: 10 December 2013

\section{References}

1. Reik W, Walter J: Genomic imprinting: parental influence on the genome. Nat Rev Genet 2001, 2:21-32.

2. Netchine I, Rossignol S, Azzi S, Le Bouc Y: Epigenetic anomalies in childhood growth disorders. Nestle Nutr Inst Workshop Ser 2013, 71:65-73.

3. Lim DH, Maher ER: Human imprinting syndromes. Epigenomics 2009, 1:347-369.

4. Reik W, Brown KW, Schneid H, Le Bouc Y, Bickmore W, Maher ER: Imprinting mutations in the Beckwith-Wiedemann syndrome suggested by altered imprinting pattern in the IGF2-H19 domain. Hum Mol Genet 1995, 4:2379-2385.

5. Gicquel C, Rossignol S, Cabrol S, Houang M, Steunou V, Barbu V, Danton F, Thibaud N, Le Merrer M, Burglen L, Bertrand AM, Netchine I, Le Bouc Y: Epimutation of the telomeric imprinting center region on chromosome 11 p15 in Silver-Russell syndrome. Nat Genet 2005, 37:1003-1007.

6. Engel JR, Smallwood A, Harper A, Higgins MJ, Oshimura M, Reik W, Schofield PN, Maher ER: Epigenotype-phenotype correlations in Beckwith-Wiedemann syndrome. J Med Genet 2000, 37:921-926.

7. Bliek J, Verde G, Callaway J, Maas SM, De Crescenzo A, Sparago A, Cerrato F, Russo S, Ferraiuolo S, Rinaldi MM, Fischetto R, Lalatta F, Giordano L, Ferrari P, Cubellis MV, Larizza L, Temple IK, Mannens MM, Mackay DJ, Riccio A: Hypomethylation at multiple maternally methylated imprinted regions including PLAGL1 and GNAS loci in Beckwith-Wiedemann syndrome. Eur J Hum Genet 2009, 17:611-619.

8. Rossignol S, Steunou V, Chalas C, Kerjean A, Rigolet M, Viegas-Pequignot E, Jouannet $P$, Le Bouc $Y$, Gicquel $C$ : The epigenetic imprinting defect of patients with Beckwith-Wiedemann syndrome born after assisted reproductive technology is not restricted to the 11 p15 region. J Med Genet 2006, 43:902-907.

9. Halliday J, Oke K, Breheny S, Algar E, Amor DJ: Beckwith-Wiedemann syndrome and IVF: a case-control study. Am J Hum Genet 2004, 75:526-528.

10. Lim D, Bowdin SC, Tee L, Kirby GA, Blair E, Fryer A, Lam W, Oley C, Cole T, Brueton LA, Reik W, Macdonald F, Maher ER: Clinical and molecular genetic features of Beckwith-Wiedemann syndrome associated with assisted reproductive technologies. Hum Reprod 2009, 24:741-747.

11. Maher ER, Brueton LA, Bowdin SC, Luharia A, Cooper W, Cole TR, Macdonald F, Sampson JR, Barratt CL, Reik W, Hawkins MM: BeckwithWiedemann syndrome and assisted reproduction technology (ART). J Med Genet 2003, 40:62-64.

12. Cooper WN, Curley R, Macdonald F, Maher ER: Mitotic recombination and uniparental disomy in Beckwith-Wiedemann syndrome. Genomics 2007, 89:613-617.

13. McRonald FE, Morris MR, Gentle D, Winchester L, Baban D, Ragoussis J, Clarke NW, Brown MD, Kishida T, Yao M, Latif F, Maher ER: CpG methylation profiling in VHL related and VHL unrelated renal cell carcinoma. Mo/ Cancer 2009, 8:31.

14. Horike S, Mitsuya K, Meguro M, Kotobuki N, Kashiwagi A, Notsu T, Schulz TC, Shirayoshi Y, Oshimura M: Targeted disruption of the human LIT1 locus defines a putative imprinting control element playing an essential role in Beckwith-Wiedemann syndrome. Hum Mol Genet 2000, 9:2075-2083.

15. Bell AC, Felsenfeld G: Methylation of a CTCF-dependent boundary controls imprinted expression of the Igf2 gene. Nature 2000, 405:482-485.

16. Mackay DJ, Callaway JL, Marks SM, White HE, Acerini $C L$, Boonen SE, Dayanikli P, Firth HV, Goodship JA, Haemers AP, Hahnemann JM, Kordonouri O, Masoud AF, Oestergaard E, Storr J, Ellard S, Hattersley AT, Robinson DO, Temple IK: Hypomethylation of multiple imprinted loci in individuals with transient neonatal diabetes is associated with mutations in ZFP57. Nat Genet 2008, 40:949-951.

17. Murdoch S, Djuric U, Mazhar B, Seoud M, Khan R, Kuick R, Bagga R, Kircheisen R, Ao A, Ratti B, Hanash S, Rouleau GA, Slim R: Mutations in NALP7 cause recurrent hydatidiform moles and reproductive wastage in humans. Nat Genet 2006, 38:300-302.

18. Meyer E, Lim D, Pasha S, Tee LJ, Rahman F, Yates JR, Woods CG, Reik W, Maher ER: Germline mutation in NLRP2 (NALP2) in a familial imprinting disorder (Beckwith-Wiedemann syndrome). PLoS Genet 2009, 5:e1000423.

19. Gicquel C, Gaston V, Mandelbaum J, Siffroi JP, Flahault A, Le Bouc Y: In vitro fertilization may increase the risk of Beckwith-Wiedemann syndrome related to the abnormal imprinting of the KCN1OT gene. Am J Hum Genet 2003, 72:1338-1341.

20. Manipalviratn SA, DeCherney A, Segars J: Imprinting disorders and assisted reproductive technology. Fertil Steril 2009, 91:305-315.

21. Owen $\mathrm{CM}$, Segars $\mathrm{JH} \mathrm{Jr}$ : Imprinting disorders and assisted reproductive technology. Semin Reprod Med 2009, 27:417-428.

22. Sutcliffe AG, Peters CJ, Bowdin S, Temple K, Reardon W, Wilson L, Clayton-Smith J, Brueton LA, Bannister W, Maher ER: Assisted reproductive therapies and imprinting disorders-a preliminary British survey. Hum Reprod 2006, 21:1009-1011.

23. Dias RP, Bogdarina I, Cazier JB, Buchanan C, Donaldson MC, Johnston LB, Hokken-Koelega AC, Clark AJ: Multiple segmental uniparental disomy associated with abnormal DNA methylation of imprinted Loci in silverrussell syndrome. J Clin Endocrinol Metab 2012, 97:E2188-E2193.

24. Azzi S, Rossignol S, Steunou V, Sas T, Thibaud N, Danton F, Le Jule M, Heinrichs C, Cabrol S, Gicquel C, Le Bouc Y, Netchine I: Multilocus methylation analysis in a large cohort of 11p15-related foetal growth disorders (Russell Silver and Beckwith Wiedemann syndromes) reveals simultaneous loss of methylation at paternal and maternal imprinted loci. Hum Mol Genet 2009, 18:4724-4733.

25. Court F, Martin-Trujillo A, Romanelli V, Garin I, Iglesias-Platas I, Salafsky I, Guitart M, Perez de Nanclares G, Lapunzina P, Monk D: Genome-wide allelic methylation analysis reveals disease-specific susceptibility to multiple methylation defects in imprinting syndromes. Hum Mutat 2013, 34:595-602.

26. Poole RL, Docherty LE, Al Sayegh A, Caliebe A, Turner C, Baple E, Wakeling E, Harrison L, Lehmann A, Temple IK, Mackay DJ, International Clinical Imprinting Consortium: Targeted methylation testing of a patient cohort broadens the epigenetic and clinical description of imprinting disorders. Am J Med Genet A 2013, 161:2174-2182.

27. Hiura H, Okae H, Miyauchi N, Sato F, Sato A, Van De Pette M, John RM, Kagami M, Nakai K, Soejima H, Ogata T, Arima T: Characterization of DNA methylation errors in patients with imprinting disorders conceived by assisted reproduction technologies. Hum Reprod 2012, 27:2541-2548.

28. Docherty LE, Kabwama S, Lehmann A, Hawke E, Harrison L, Flanagan SE, Ellard S, Hattersley AT, Shield JP, Ennis S, Mackay DJ, Temple IK: Clinical presentation of 6q24 transient neonatal diabetes mellitus (6q24 TNDM) and genotype-phenotype correlation in an international cohort of patients. Diabetologia 2013, 56:758-762.

29. Elliott M, Bayly R, Cole T, Temple IK, Maher ER: Clinical features and natural history of Beckwith-Wiedemann syndrome: presentation of 74 new cases. Clin Genet 1994, 46:168-174.

30. Abu-Amero S, Monk D, Frost J, Preece M, Stanier P, Moore GE: The genetic aetiology of Silver-Russell syndrome. J Med Genet 2008, 45:193-199.

31. Lefebvre L, Viville S, Barton SC, Ishino F, Keverne EB, Surani MA: Abnormal maternal behaviour and growth retardation associated with loss of the imprinted gene mest. Nat Genet 1998, 20:163-169. 
32. Foulstone E, Prince S, Zaccheo O, Burns JL, Harper J, Jacobs C, Church D, Hassan AB: Insulin-like growth factor ligands, receptors, and binding proteins in cancer. J Pathol 2005, 205:145-153.

33. Xu Y, Goodyer CG, Deal C, Polychronakos C: Functional polymorphism in the parental imprinting of the human IGF2R gene. Biochem Biophys Res Commun 1993, 197:747-754.

34. Young LE, Fernandes K, McEvoy TG, Butterwith SC, Gutierrez CG, Carolan C, Broadbent PJ, Robinson JJ, Wilmut I, Sinclair KD: Epigenetic change in IGF2R is associated with fetal overgrowth after sheep embryo culture. Nat Genet 2001, 27:153-154.

35. Turner CL, Mackay DM, Callaway JL, Docherty LE, Poole RL, Bullman H, Lever M, Castle BM, Kivuva EC, Turnpenny PD, Mehta SG, Mansour S, Wakeling EL, Mathew V, Madden J, Davies JH, Temple IK: Methylation analysis of 79 patients with growth restriction reveals novel patterns of methylation change at imprinted loci. Eur J Hum Genet 2010, 18:648-655.

36. Buiting K, Gross S, Lich C, Gillessen-Kaesbach G, El-Maarri O, Horsthemke B: Epimutations in Prader-Willi and Angelman syndromes: a molecular study of 136 patients with an imprinting defect. Am J Hum Genet 2003, 72:571-577.

37. Bens S, Haake A, Richter J, Leohold J, Kolarova J, Vater I, Riepe FG, Buiting K, Eggermann T, Gillessen-Kaesbach G, Platzer K, Prawitt D, Caliebe A, Siebert $R$ : Frequency and characterization of DNA methylation defects in children born SGA. Eur J Hum Genet 2013, 21:838-843.

38. Amor DJ, Halliday J: A review of known imprinting syndromes and their association with assisted reproduction technologies. Hum Reprod 2008, 23:2826-2834.

39. Varrault A, Gueydan C, Delalbre A, Bellmann A, Houssami S, Aknin C, Severac D, Chotard L, Kahli M, Le Digarcher A, Pavlidis P, Journot L: Zac1 regulates an imprinted gene network critically involved in the control of embryonic growth. Dev Cell 2006, 11:711-722.

40. Zhao Z, Tavoosidana G, Sjölinder M, Göndör A, Mariano P, Wang S, Kanduri C, Lezcano M, Sandhu KS, Singh U, Pant V, Tiwari V, Kurukuti S, Ohlsson R: Circular chromosome conformation capture (4C) uncovers extensive networks of epigenetically regulated intra- and interchromosomal interactions. Nat Genet 2006, 38:1341-1347.

doi:10.1186/1868-7083-5-23

Cite this article as: Tee et al:: Epimutation profiling in Beckwith-Wiedemann syndrome: relationship with assisted reproductive technology. Clinical Epigenetics 2013 5:23.

\section{Submit your next manuscript to BioMed Central and take full advantage of:}

- Convenient online submission

- Thorough peer review

- No space constraints or color figure charges

- Immediate publication on acceptance

- Inclusion in PubMed, CAS, Scopus and Google Scholar

- Research which is freely available for redistribution 